\title{
Gerontology
}

\section{Aging and Regional Differences in Fat Cell Progenitors - A Mini-Review}

\author{
Anna Sepe $^{\mathrm{a}, \mathrm{b}}$ Tamara Tchkonia $^{\mathrm{b}}$ Thomas Thomou $^{\mathrm{b}}$ Mauro Zamboni $^{\mathrm{a}}$ \\ James L. Kirkland ${ }^{a}, b$ \\ aDepartment of Biomedical and Surgical Sciences, Division of Geriatrics, University of Verona, Verona, Italy; \\ ${ }^{\mathrm{b}}$ Mayo Clinic Robert and Arlene Kogod Center on Aging, Rochester, Minn., USA
}

\section{Key Words}

Preadipocytes - Fat redistribution • Fat depot •

Inflammation

\begin{abstract}
Fat mass and fat tissue distribution change dramatically throughout life. In old age, fat becomes dysfunctional and is redistributed from subcutaneous to intra-abdominal visceral depots as well as other ectopic sites, including bone marrow, muscle and the liver. These changes are associated with increased risk of metabolic syndrome. Fat tissue is a nutrient storage, endocrine and immune organ that undergoes renewal throughout the lifespan. Preadipocytes, which account for $15-50 \%$ of cells in fat tissue, give rise to new fat cells. With aging, declines in preadipocyte proliferation and differentiation likely contribute to increased systemic exposure to lipotoxic free fatty acids. Age-related fat tissue inflammation is related to changes that occur in preadipocytes and macrophages in a fat depot-dependent manner. Fat tissue inflammation frequently leads to further reduction in adipogenesis with aging, more lipotoxicity and activation of cellular stress pathways that, in turn, exacerbate inflammatory responses of preadipocytes and immune cells, establishing self-perpetuating cycles that lead to systemic dysfunction. In this review, we will consider how inherent,
\end{abstract}

age-related, depot-dependent alterations in preadipocyte function contribute to age-related fat tissue redistribution and metabolic dysfunction.

Copyright ๑ 2010 S. Karger AG, Basel

\section{Introduction}

Some studies project that one third of the world's population will be over 60 years of age by the end of this century [1]. Concurrently, there has been a marked increase in the prevalence of overweight and obese individuals in developed countries, affecting principally younger and middle-aged adults. Sixty-six percent of US adults are either overweight or obese [2]. In advanced old age, while the total amount of fat tissue tends to decline or remains stable, fat distribution changes dramatically. By advanced old age, fat is redistributed from subcutaneous to intraabdominal visceral depots and to ectopic sites, including muscle, liver and bone marrow [3-5]. Fat redistribution in the elderly is associated with an increased risk for metabolic syndrome (diabetes, hypertension, dyslipidemia, atherosclerosis and relatively increased intra-abdominal fat $[3,4,6,7])$.

Both central fat redistribution and metabolic disorders are more common in elderly than younger women 
[3]. Moreover, visceral fat content and intramuscular lipids are markers of dysregulated insulin responsiveness independent of total body obesity, and are associated with substantially increased prevalence of metabolic syndrome $[6,7]$. These studies suggest that insulin resistance and altered glucose homeostasis are more closely related to regional adipose tissue distribution than total fat mass. Indeed, the relation between visceral fat content and glucose intolerance is even stronger in normal weight men and women than in overweight or obese men and women [7].

While excess intra-abdominal fat tissue is independently correlated with insulin resistance $[8,9]$, subcutaneous fat tissue dysfunction, leading to inability to store excess energy, may result in expansion of potentially deleterious visceral fat and metabolic disease. This is associated with ectopic fat deposition in liver, skeletal muscle, heart, and pancreatic $\beta$-cells [10]. These changes appear to occur in both old age and in lipodystrophies.

As in advanced old age, patients with certain lipodystrophies have selective loss of subcutaneous adipose tissue as well as intra-abdominal fat expansion [11]. They develop insulin resistance and dyslipidemia with marked hypertriglyceridemia, mild hypercholesterolemia and reduced high-density lipoprotein cholesterol. Glycerol levels and free fatty acid turnover are increased without increased free fatty acid oxidation. Mutations of genes that encode lipodystrophic syndromes (1-acylglycerol-3phosphate-O-acyltransferase 2, Berardinelli-Seip congenital lipodystrophy 2 , caveolin 1 , lamin $\mathrm{A} / \mathrm{C}$, peroxisome proliferator-activated receptor- $\gamma$ or v-Akt murine thymoma oncogene homolog 2) may do so by impairing triglyceride synthesis or storage. Consistent with this, a recent study in transgenic rats with a mutation of the lamin $\mathrm{A} / \mathrm{C}$ gene indicated impaired differentiation of preadipocytes into mature adipocytes [12]. Interestingly, this is the same gene that causes Hutchinson-Guilford progeria, a condition also associated with subcutaneous fat loss. It is still not clear why the loss of fat tissue is sitespecific in lipodystrophies and does not uniformly involve all fat depots.

Fat tissue is an endocrine organ capable of expressing and secreting many different autocrine, paracrine and endocrine factors [13]. Fat tissue regulates a range of processes that affect metabolic function, including adipogenesis, glucose metabolism, production of components of the renin-angiotensin system, and enzyme and hormone production and processing as well as angiogenesis, immune responses and hemostasis. It releases chemokines and cytokines that can contribute to metabolic dys- function and attract pro-inflammatory $\mathrm{T}$ lymphocytes, mast cells and macrophages.

Macrophage abundance is often increased in adipose tissue of both obese and lipodystrophic subjects [14-20]. Infiltration with activated pro-inflammatory $\mathrm{T}$ lymphocyte subsets and mast cells and loss of immunomodulatory and anti-inflammatory $\mathrm{T}$ lymphocyte subtypes occur before macrophage infiltration in obesity and may contribute to macrophage infiltration and activation [2125]. Activated macrophages, in turn, exacerbate the fat tissue pro-inflammatory state and insulin resistance. Indeed, macrophages in lipodystrophic HIV-infected subjects may be involved in adipose tissue wasting $[19,26]$. Macrophage infiltration and recruitment in HIV-related lipodystrophy are closely related to anti-retroviral drug treatment $[19,20]$. This occurs in a fat-depot specific pattern [26]. Dysfunctional fat tissue in obese individuals may promote inflammation due to enlarged adipocytes that produce inflammatory cytokines. Even if the precise details of the pathophysiology of the fat tissue damage are different in obesity from the lipodystrophies, the resulting metabolic dysfunction is strikingly similar. However, neither the hypothesis that metabolic dysfunction is due to impaired lipid storage causing lipotoxicity, nor that dysfunction is due to infiltration by immune effectors, completely explain what happens in old age. Age-related fat tissue dysfunction may be related to a combination of mechanisms, with reduced capacity to store lipotoxic fatty acids, fat tissue damage by fatty acids and inflammation being contributing factors. These processes are interrelated and amplify each other. The potential contributions of inherent age-related dysfunction of adipocytes, and particularly of the progenitors from different fat depots that give rise to new fat tissue throughout life, to agerelated changes in fat tissue are addressed below.

\section{The Adipose Organ and Aging}

The main role of fat tissue is to store energy, which is concentrated in neutral triglycerides. Triglycerides may arise from de novo lipogenesis, with synthesis of triglycerides from non-lipid precursors, or hydrolysis of circulating lipids, uptake as free fatty acids and re-esterification into triglycerides. These intracellular triglycerides can be rapidly hydrolyzed by lipases into glycerol and free fatty acids that are then released and transported to other tissues to be oxidized in mitochondria. It has been reported that mobilization of lipids is impaired in old mice due to abnormalities in gene expression for apolipopro- 
teins. In response to stressful external stimuli (such as fasting), young mice maintain normal energy homeostasis by activating lipoprotein lipase, while old mice are largely unable to adapt to such challenges [27]. However, in the face of positive caloric balance, if adipogenesis is impaired and intracellular adipocyte hydrolysis of triglycerides exceeds intracellular free fatty acid esterification, there is a net release of free fatty acids into the circulation. This, if extensive enough, contributes to systemic metabolic dysfunction [28]. Increased fatty acid delivery to other organs, especially when coupled with defective fatty acid oxidation, leads to ectopic triglyceride storage in these organs, contributing to systemic dysfunction. Cytotoxic effects of fatty acids are termed lipotoxicity [13]. Saturated fatty acids, including palmitate and stearate, are more lipotoxic than unsaturated fatty acids, such as palmitoleate, oleate or linoleate, possibly related to the ceramide generated by saturated fatty acids [29]. Palmitate, oleate and linoleate are the most common fatty acids in the human diet as well as in human and animal fat tissue.

Small fat cells defend against lipotoxicity by sequestering excess fatty acids as neutral triglycerides. Preadipocytes, which account for $15-50 \%$ of the cells in fat tissue, give rise to new fat cells throughout life as fat tissue turns over, and also defend against lipotoxicity. However, with aging, even preadipocytes become increasingly susceptible to lipotoxicity [30]. Preadipocytes from old rodents treated with oleate accumulated lipid in multiple small droplets characteristic of lipotoxicity, developed morphological changes in their nuclei and had increased caspase activity, consistent with increased apoptotic activity. Furthermore, preadipocytes from old rodents had lower differentiation-dependent gene expression than preadipocytes from young animals treated with oleate. Reduced capacity of preadipocytes to express differentiation-dependent proteins that bind cytotoxic fatty acids and convert them into less cytotoxic triglycerides with aging may, in turn, diminish defense against lipotoxicity, setting up a vicious cycle that accelerates preadipocyte and fat tissue dysfunction.

Saturated fatty acids, such as palmitate, are particularly lipotoxic. In the absence of inducers of preadipocyte differentiation, palmitate induces stress responses and proapoptotic transcription factor expression in preadipocytes [31]. Treatment with oleate or linoleate reverses this palmitate-induced apoptosis. The negative impact of palmitate on fat cells has been demonstrated in several in vitro studies [31-35]. Furthermore, saturated fatty acids are involved in fat tissue inflammation. In vitro studies
[32-35] indicated that 3T3-L1 adipocytes preloaded with saturated fatty acids, but not with unsaturated fatty acids, exhibited oxidative stress and expression of pro-inflammatory cytokines, including monocyte chemoattractant protein-1 (MCP-1, a macrophage chemokine), tumor necrosis factor- $\alpha$ (TNF- $\alpha$ ) and interleukin-6. Palmitatetreated 3T3-L1 adipocytes had increased MCP-1 [33]. Moreover, exogenously administered TNF- $\alpha$ enhanced MCP-1 release from adipocytes. Increased adipocyte TNF- $\alpha$ release was also found in co-culture studies of adipocytes with macrophages $[34,35]$. Saturated fatty acids, including palmitate and laurate, induced TNF- $\alpha$ expression in macrophages, which, in turn, promoted lipolysis and augmented adipocyte MCP-1 expression. These inflammatory changes were evident in co-culture without direct contact between cells, indicating that adipocytes and macrophages communicate at least in part through paracrine mechanisms. Furthermore, fat tissue from obese subjects induces $\mathrm{T}$ lymphocyte responses leading to interferon- $\gamma$ release and macrophage activation [22-25]. Thus, saturated fatty acids may be especially deleterious to fat tissue function in old age, since preadipocytes become paradoxically susceptible to lipotoxicity with aging, and palmitate is even lipotoxic in preadipocytes and fat cells from younger individuals.

Mesenchymal progenitors are thought to be self-replicating and capable of differentiating into mature adipocytes and other mesenchymal cell types. Upon exposure to nutrients, hormonal effectors (such as insulin, glucocorticoids, insulin-like growth factor-1), and paracrine and autocrine effectors (including free fatty acids and cyclic AMP), mesenchymal progenitor-derived preadipocytes begin to express transcription factors [including proliferator activated receptor- $\gamma$ (PPAR- $\gamma$ ) and the CCAAT/enhancer binding protein (C/EBP) family] and differentiate into mature fat cells $[10,36]$. In addition to preadipocytes, mesenchymal progenitors can give rise to bone, cartilage and muscle progenitors, as well as macrophages and renal mesangial cells [37]. Cellular stress responses activated by inflammatory cytokines, lipotoxicity and inherent age-related changes in progenitor function may cause dysdifferentiation of mesenchymal progenitors into partially differentiated, adipocyte-like cells [mesenchymal adipocyte-like default cells (MAD cells); [37]]. Indeed, MAD cells with a partial fat cell-like phenotype and expression of genes usually expressed in fat (e.g. PPAR- $\gamma$ ) develop from muscle progenitors (muscle satellite cells) and bone marrow progenitors cultured from old individuals [37]. These MAD cells may contribute to accumulation of fat in non-adipose tissues with ag- 
Fig. 1. Human preadipocyte capacity for lipid accumulation declines with age. Preadipocytes isolated from subcutaneous abdominal fat derived from healthy young (26 years, BMI: 27.6, male) and old subjects (79 years, BMI: 29.1, male), and passaged for four population doublings until confluency, were treated with differentiationinducing medium for 7 days.

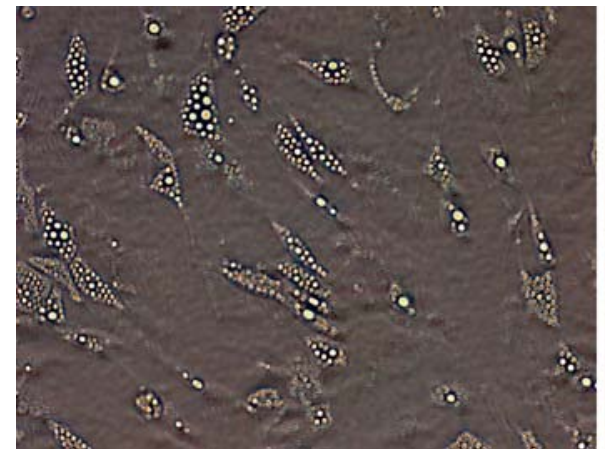

26 years

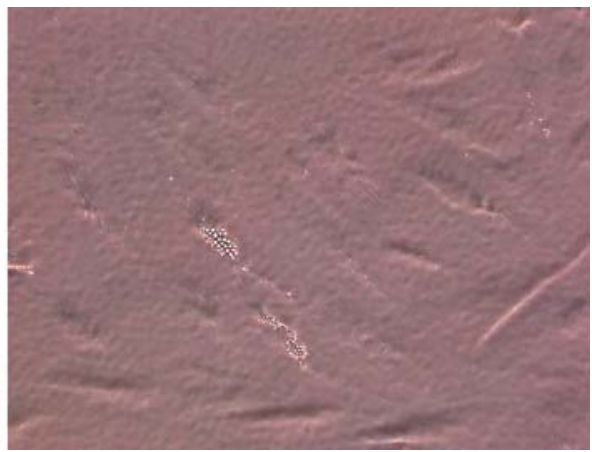

79 years ing. Even preadipocytes from old individuals behave like MAD cells, as they are not capable of differentiating into fully functional fat cells [37].

The fat tissue deregulation that occurs in aging, obesity and lipodystrophy may initiate self-propagating inflammatory cycles. Dysfunctional fat cells start to secrete pro-inflammatory cytokines and chemokines that, in turn, alter T lymphocyte subsets, attract mast cells and cause monocyte recruitment and macrophage activation. Factors secreted by macrophages induce the release of fatty acids from adipocytes that impede differentiation of preadipocytes into fat cells and cause dysdifferentiation of mesenchymal progenitors into MAD cells. All these processes contribute to lipotoxicity in adipose tissue and other organs, elicit cellular stress responses, cause even more generation of inflammatory cytokines and chemokines, further block adipogenesis, and result in further release of lipotoxic fatty acid [13].

\section{Adipogenesis and Aging}

Preadipocyte capacities for replication and differentiation decline with aging $[36,38,39]$. The decline in fat depot size with aging is not due to a decrease in adipocyte number, but rather to decreased adipocyte size [40, 41]. This suggests that new fat cells are formed throughout one's life span. In old age, fat cell progenitors are not as capable of lipid accumulation or insulin responses as cells from younger individuals, likely contributing to the increased abundance of small, insulin-resistant, dysfunctional fat cells with aging. We found that preadipocytes isolated from old humans and treated with differentiation-inducing medium accumulated fewer doubly refractile lipid inclusions than preadipocytes from young subjects [39] (fig. 1). Glycerol-3-phosphate dehydrogenase, a differentiation-dependent gene product, decreases with aging in humans [42]. Decreased capacity for differentiation with increased age is at least partly due to decreased expression of key transcription factors involved in adipogenesis. Transcription factors in the C/EBP family, as well as PPAR- $\gamma$, have important roles in the differentiation program. They act sequentially in a signaling cascade that leads to the fat cell phenotype. C/EBP- $\beta$ and $-\delta$ are expressed first, followed by C/EBP- $\alpha$ and PPAR- $\gamma$. Once $\mathrm{C} / \mathrm{EBP}-\alpha$ is expressed, it maintains expression of PPAR- $\gamma$ and C/EBP- $\alpha$ itself [43]. The process of adipocyte differentiation is restrained by anti-adipogenic factors, including inhibitory C/EBP family members, such as C/EBP- $\beta$ liver inhibitory protein (C/EBP- $\beta$-LIP) and C/EBP homologous protein (CHOP). They form heterodimers with adipogenic C/EBP family members or prevent adipogenic C/EBP family members from binding to differentiation-dependent promoters, inhibiting adipogenesis. CUG triplet repeat RNA binding protein (CUGBP) is upstream of C/EBP- $\beta$-LIP. CUGBP binds to C/EBP- $\beta$ mRNA, resulting in switching from translation of the adipogenic C/EBP- $\beta$ LAP to the anti-adipogenic C/EBP- $\beta$-LIP protein isoform.

We found that expression of C/EBP- $\alpha$ declines with aging in differentiating primary rat preadipocytes and fat cells [39]. Increasing C/EBP- $\alpha$ expression by transfecting cells derived from old rats partially restored their capacity to differentiate into fat cells. This suggests that downstream, differentiation-dependent genes retain their ability to respond to overexpressed levels of C/EBP- $\alpha$. Thus, the mechanisms causing inherently impaired adipogenesis with aging act at or before the level of adipogenic transcription factor expression during differentiation.

Anti-adipogenic C/EBP- $\beta$-LIP increases both in cultured differentiating preadipocytes from different fat depots and in fat tissue in vivo. This could impact down- 
stream differentiation-dependent genes, including those involved in glucose homeostasis and lipid metabolism. We found that increased C/EBP- $\beta$-LIP is related to an increase in CUGBP with aging [44]. Inhibiting CUGBP in preadipocytes from old animals led to decreased $\mathrm{C} /$ EBP- $\beta$-LIP and enhanced lipid accumulation in these cells. Furthermore, TNF- $\alpha$, which is secreted to a greater extent in fat tissue from old individuals than from younger individuals, increased preadipocyte CUGBP activity. TNF- $\alpha$ also enhances CHOP expression in primary rat preadipocytes. CHOP, in turn, is anti-adipogenic. TNF- $\alpha$ short interference RNA reduced $\mathrm{CHOP}$ expression and partially restored lipid accumulation [45]. TNF- $\alpha$ inhibits insulin action through a variety of mechanisms [46], also contributing to its anti-adipogenic effects. TNF- $\alpha$ secretion varies not only with aging, but among different fat depots as well. In rats, epididymal preadipocytes release more TNF- $\alpha$ than perirenal cells, consistent with the finding that epididymal preadipocytes have lower capacity for lipid accumulation and less complete differentiation than perirenal cells [33].

\section{Regional Variation in Fat Cell Progenitor Function}

Human studies provide support for the hypothesis that preadipocytes and fat cells from different regions are inherently distinct. Genome-wide expression profiles of primary preadipocytes from human abdominal subcutaneous, mesenteric and omental fat tissue differed with respect to gene signatures [47]. Mesenteric preadipocytes had gene expression profiles closer to those of subcutaneous than to omental cells. Many of these differentiallyexpressed genes are involved in lipid metabolism. The most prominent distinct class of genes were developmental regulators, indicating preadipocytes from different fat depots are distinct cell subtypes $[47,48]$.

We found human abdominal subcutaneous preadipocytes had the highest capacity to differentiate, as indicated by lipid accumulation and expression of the fat cell fatty acid binding protein, FABP- 4 , as well as PPAR- $\gamma$ and C/EBP- $\alpha$. Mesenteric preadipocytes from the same subjects had intermediate capacity for adipogenesis and omental cells had the lowest. Transfection with C/EBP- $\alpha$ enhanced capacity of omental preadipocytes to accumulate lipid, suggesting that genes upstream of $\mathrm{C} / \mathrm{EBP}-\alpha$ contribute to regional differences in fat depots. Differences in preadipocyte replication and differentiation remained evident even in colonies derived from single primary subcutaneous compared to mesenteric or omental preadipocytes [47, 49]. Depot-dependent differences in preadipocyte replication, differentiation and susceptibility to TNF- $\alpha$-induced apoptosis and developmental gene expression profiles remained remarkably similar in primary preadipocyte cultures and in preadipocyte strains created by stably expressing human telomere reverse transcriptase in clones derived from single human abdominal subcutaneous, mesenteric and omental preadipocytes after 40 population doublings $[47,49]$. These findings indicate that the distinct properties of fat cell progenitors from different fat depots are inherent. Van Harmelen et al. [50] also reported that subcutaneous preadipocytes proliferated at a greater rate than omental cells, but did not find regional variation in capacity for differentiation, which could be related to differences in the differentiation-inducing media used. Van Harmelen and colleagues also found that age correlated negatively with proliferation only in subcutaneous preadipocytes and not in the omental depot. This confirms that aging has distinct effects on preadipocytes from different fat depots and could explain loss of subcutaneous and relative preservation of omental fat with aging.

\section{Fat Inflammation and Aging}

Adipose tissue produces mediators of inflammation and insulin resistance. Preadipocytes likely play a key and underappreciated role in the pathogenesis of inflammation. Recent reports show that pro-inflammatory cytokines and chemokines are expressed predominantly in preadipocytes, rather than in adipocytes [51, 52]. Chung et al. [51] reported that human preadipocytes co-cultured with adipocytes (in the absence of macrophages) produce TNF- $\alpha$, interleukin- 6 and MCP-1 in response to lipopolysaccharide (LPS). Expression of these inflammatory mediators decreased as the degree of differentiation progressed. After LPS treatment, preadipocyte expression of adiponectin and PPAR- $\gamma$, which are associated with insulin sensitivity, was attenuated. This suggests that stressed preadipocytes may initiate lymphocyte subset, mast cell and macrophage recruitment in adipose tissue and promote inflammatory responses that lead to impaired adipogenesis, insulin resistance and metabolic dysfunction.

Toll-like receptors (TLR) are components of the pathway through which LPS causes expression of its downstream pro-inflammatory targets. TLRs are a family of receptors that are critical in the innate immune response to foreign pathogens and micro-organisms. TLR4 is con- 
stitutively expressed in both preadipocytes and adipocytes $[34,51,53]$. In contrast, TLR2 expression is robustly induced by LPS only in preadipocytes and may, in part, be responsible for the greater inflammatory responsiveness of preadipocytes than fat cells [51]. Stimulation of TLRs initiates intracellular signaling cascades resulting in downstream NFкB- and mitogen-activated protein kinase activation and upregulation of cytokine release. $\mathrm{NF \kappa B}$ is an inducible transcription factor that plays a central role in regulation of inflammation. Its inhibition leads to decreasing inflammatory responses [32]. Saturated fatty acids, which can be released by fat cells upon interaction with macrophages, activate $\mathrm{NF \kappa B}$ in macrophages through TLR4 $[34,35]$. Furthermore, studies involving co-culture of preadipocytes and adipocytes indicate that preadipocytes can mediate insulin resistance in adipocytes [51]. From these observations it can be inferred that preadipocytes play a major role as initiators of inflammation, promoting a cascade of events that results in the transmission of paracrine signals to neighboring adipocytes; recruitment of inflammatory $\mathrm{T}$ lymphocyte subsets, mast cells and monocytes from the blood; activation of resident macrophages; and impairment in fat cell insulin responsiveness [51]. At least in obesity and perhaps with aging, cross-talk among these different cell types in fat tissue leads to a pro-inflammatory state that may contribute to systemic metabolic dysfunction [13, 32].

Adipose tissue of obese animals and humans becomes infiltrated by macrophages [14-18]. Macrophage content correlates strongly with the extent of obesity and fat redistribution [17, 18]. In obesity, both experimental animals and humans accumulate more macrophages in visceral fat than subcutaneous fat $[17,54]$. Different theories about the mechanism by which macrophages are recruited in obesity have been proposed. Visceral fat depots exhibit more extensive cell death in their adipocyte population as opposed to subcutaneous depots [54]. This is in accord with our finding that preadipocyte telomeraseexpressing strains derived from human visceral preadipocytes (and thus devoid of the presence of other cell types including macrophages) are more susceptible to apoptosis in response to TNF- $\alpha$ than subcutaneous telomerase-expressing strains [49]. It appears that chemokines secreted from preadipocytes contribute to fat tissue inflammation in obesity. Increased secretion of MCP-1 and other factors by preadipocytes could initiate $\mathrm{T}$ lymphocyte, mast cell and macrophage recruitment and activation in obesity.

Preadipocytes and Aging
How age-associated changes in subcutaneous and visceral fat are related to macrophage recruitment and systemic inflammation are poorly understood. A recent study [18] indicated that in younger animals, the highest percentage of macrophages is in visceral fat. Their numbers remain high with advancing age. Conversely, subcutaneous macrophage abundance was initially low but increased with aging. Since subcutaneous fat is 10 to 20 times more abundant than intra-abdominal visceral fat, this could have important systemic consequences. This contrasts with the report of Harman-Boehm et al. [17], in which a significant positive association between age and percent of macrophages in human omental adipose tissue was observed. However, little variation in macrophage content in subcutaneous fat tissue was found. While more work needs to be done, there are strong indications that fat tissue macrophage abundance changes with aging and state of obesity, and is depot-dependent, linking dysfunctional adipose tissue to inflammation and systemic dysfunction. Much less is known about fat tissue T lymphocyte and mast cell abundance with aging and among fat depots.

Fat tissue inflammation with aging appears to be caused by changes in both preadipocytes and macrophages. Dysfunctional preadipocytes release more proinflammatory cytokines and chemokines that induce macrophage recruitment and activation. Cytokines produced by macrophages increase preadipocyte release of cytotoxic free fatty acids. These fatty acids are distributed to different organs, contributing to ectopic fat deposition, organ dysfunction and metabolic disorders, all of which become increasingly common in old age. Very little is known about age-related changes in fat tissue macrophage function. In other types of macrophages, the capacity to be induced into an inflammatory state actually declines with aging [55]. If fat tissue macrophage function also decreases with aging, this would indicate a greater role for other cell types, such as activated preadipocytes, in causing fat tissue inflammation with aging than is the case in obesity in younger subjects in whom macrophage responsiveness is intact.

\section{Conclusions}

Aging is characterized by body fat redistribution with increased visceral fat and relative loss of subcutaneous fat, especially in the periphery. The impaired capacity of fat tissue to store lipids associated with aging may be responsible for increased systemic free fatty acid exposure, 
Fig. 2. Potential depot-dependent differences in age-related changes in fat tissue distribution and function.

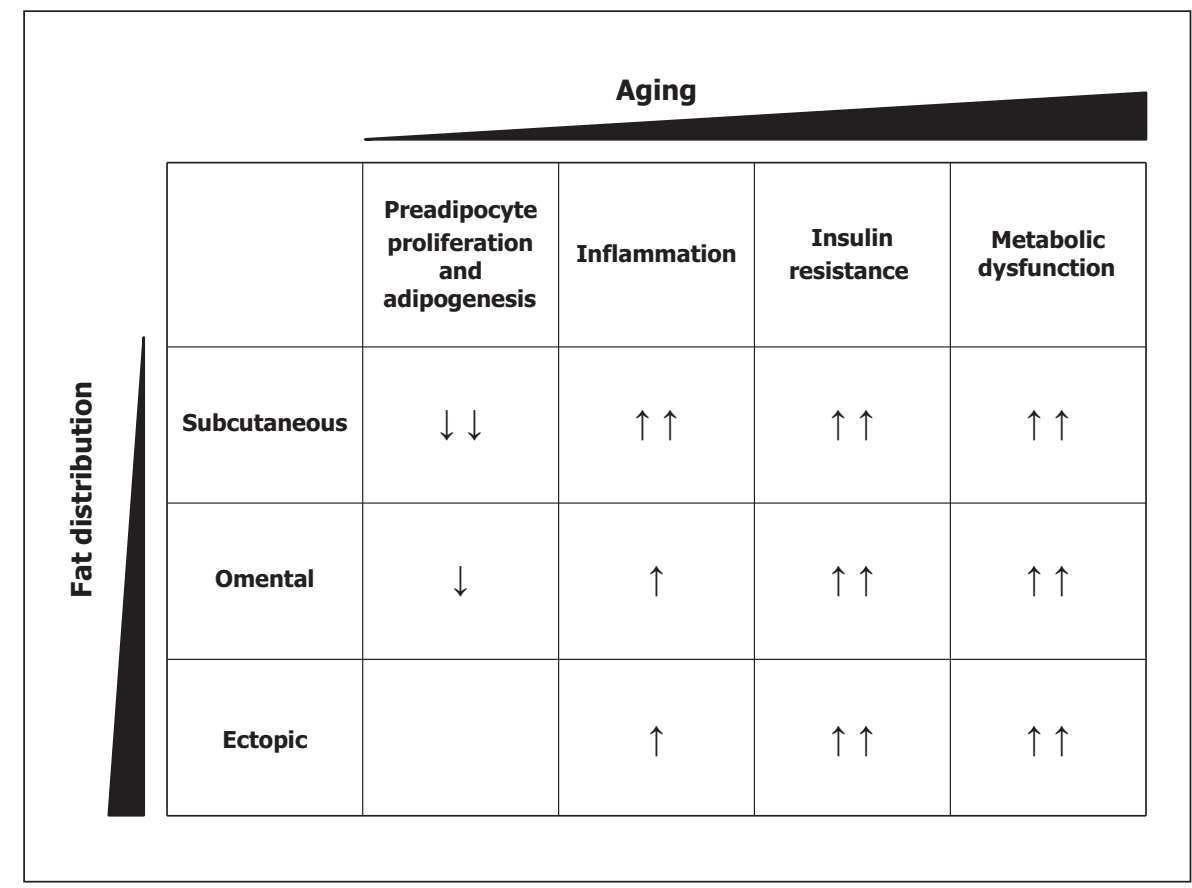

Fig. 3. Possible mechanisms of age-related mesenchymal progenitor dysfunction and ultimately fat redistribution and metabolic disease.

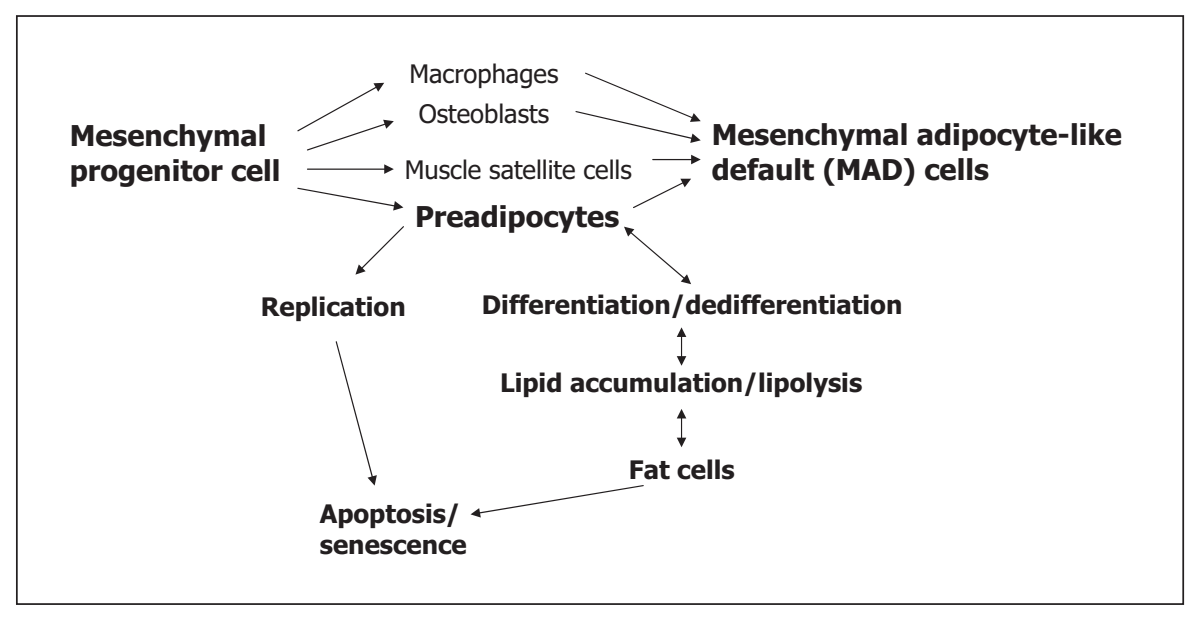

leading to ectopic fat deposition, lipotoxicity and metabolic disease. Preadipocytes become dysfunctional with aging. They become paradoxically susceptible to lipotoxicity and have reduced capacities for proliferation and differentiation, leading to impaired fatty acid handling. Saturated fatty acids are especially deleterious, exerting particularly adverse systemic effects. Free fatty acids can actually impair adipogenesis by causing lipotoxicity in preadipocytes from older individuals, stimulating lipolysis and inducing cytokine secretion in preadipocytes, macrophages and perhaps other immune effectors, lead- ing to a self-perpetuating vicious circle. Preadipocytes from old rats release more TNF- $\alpha$ than from young animals, which is amplified by the fat redistribution that occurs with aging. Epididymal preadipocytes release more TNF- $\alpha$ than perirenal cells and are more susceptible to apoptosis. Similarly, in humans omental adipocytes are more susceptible to TNF- $\alpha$-induced apoptosis than subcutaneous adipocytes. Macrophages (a major source of TNF- $\alpha$ ), at least in obesity in younger subjects. Macrophage-released TNF- $\alpha$ impedes preadipocyte differentiation in old rodents. Some reports indicate that in obe- 
sity and lipodystrophy, macrophage content is higher in visceral fat than in subcutaneous fat. However, few studies have addressed the relationship between aging and fat depot origin on the one hand and macrophage recruitment on the other. In rats with varying degrees of obesity, macrophage content increases with aging mainly in subcutaneous fat and remains high throughout life in visceral fat. These findings suggest that even subcutaneous fat, believed to be protective in young individuals, may become dysfunctional and deleterious with aging (fig. 2).

The responsible upstream mechanisms (fig. 3) and contribution of inherent preadipocyte characteristics (including reduced capacities for replication and differentiation) to fat tissue dysfunction with aging remain to be investigated in greater detail. Cellular senescence occurs together with fat tissue inflammation in obesity $[56,57]$ and in fat tissue dysfunction in mice with progeric syndromes induced by DNA-damaging mutations [58]. The role of cellular senescence in fat tissue inflammation, dysfunction, redistribution and depot-dependent trajectories with aging remains to be defined. Mechanisms responsible for regional variation in the trajectories of fat tissue dysfunction with aging are poorly understood. Interactions among different cell types, effects of fat depot origin on these relations, and their contribution to fat re- distribution and metabolic dysfunction with aging all require further investigation. Another issue that merits scrutiny is the elucidation of mechanisms responsible for differences in macrophage abundance among fat depots with aging. The finding that inhibition of TNF- $\alpha$ in preadipocytes partially restores adipogenesis indicates that pharmacological interventions based on reducing inflammatory cytokines or cellular stress responses might delay metabolic dysfunction with aging. Elucidation of the mechanisms through which different fat depots become dysfunctional with aging might open opportunities for development of clinical interventions. This is particularly important as fat tissue is at the nexus of processes involved in determination of lifespan and onset of agerelated diseases (interventions such as caloric restriction increase lifespan and delay age-related disease in multiple species, and manipulating fat tissue growth by knocking out fat tissue insulin receptors extends lifespan in rodents).

\section{Acknowledgements}

The authors are grateful for the support of the Cassa di Risparmio di Verona-Vicenza, Belluno, Ancona (Biomedical Research Project 2007), NIH grants AG013925 and AG31736 (J.L.K.), and the Ted Nash Foundation (J.L.K.).

\section{References}

1 Lutz W, Sanderson W, Scherbov S: The coming acceleration of global population ageing. Nature 2008;451:716-719.

2 Wang YC, Colditz GA, Kuntz KM: Forecasting the obesity epidemic in the aging US Population. Obesity (Silver Spring) 2007; 15: 2855-2865.

3 Zamboni M, Armellini F, Harris T, Turcato E, Micciolo R, Bergamo-Andreis IA, Bosello O: Effects of age on body fat distribution and cardiovascular risk factors in women. Am J Clin Nutr 1997;66:111-115.

4 DeNino WF, Tchernof A, Dionne IJ, Toth MJ, Ades PA, Sites CK, Poehlman ET: Contribution of abdominal adiposity to age-related differences in insulin sensitivity and plasma lipids in healthy nonobese women. Diabetes Care 2001;24:925-932.

5 Hughes VA, Roubenoff R, Wood M, Frontera WR, Evans WJ, Fiatarone Singh MA: Anthropometric assessment of $10-\mathrm{y}$ changes in body composition in the elderly. Am J Clin Nutr 2004;80:475-482.
-6 Goodpaster BH, Krishnaswami S, Resnick $\mathrm{H}$, Kelley DE, Haggerty C, Harris TB, Schwartz AV, Kritchevsky S, Newman AB: Association between regional adipose tissue distribution and both type 2 diabetes and impaired glucose tolerance in elderly men and women. Diabetes Care 2003;26:372379.

7 Goodpaster BH, Krishnaswami S, Harris TB, Katsiaras A, Kritchevsky SB, Simonsick EM, Nevitt M, Holvoet P, Newman AB: Obesity, regional body fat distribution, and the metabolic syndrome in older men and women. Arch Intern Med 2005;165:777-783.

$>8$ Kelley DE, Thaete FL, Troost F, Huwe T, Goodpaster BH: Subdivisions of subcutaneous abdominal adipose tissue and insulin resistance. Am J Physiol Endocrinol Metab 2000;278:E941-E948.

-9 Miyazaki Y, Glass L, Triplitt C, Wajcberg E, Mandarino LJ, DeFronzo RA: Abdominal fat distribution and peripheral and hepatic insulin resistance in type 2 diabetes mellitus. Am J Physiol Endocrinol Metab 2002;283: E1135-E1143.
10 Heilbronn L, Smith SR, Ravussin E: Failure of fat cell proliferation, mitochondrial function and fat oxidation results in ectopic fat storage, insulin resistance and type II diabetes mellitus. Int J Obes Relat Metab Disord 2004;28(Suppl 4):S12-S21.

11 Garg A, Agarwal AK: Lipodystrophies: disorders of adipose tissue biology. Biochim Biophys Acta 2009;1791:507-513.

12 Wojtanik KM, Edgemon K, Viswanadha S, Lindsey B, Haluzik M, Chen W, Poy G, Reitman M, Londos C: The role of LMNA in adipose: a novel mouse model of lipodystrophy based on the Dunnigan-type familial partial lipodystrophy mutation. J Lipid Res 2009;50: 1068-1079.

13 Tchkonia T, Corkey BE, Kirkland JL: Current views of the fat cell as an endocrine cell: lipotoxicity. Endocrine Updates 2006;26: 105-118.

14 Weisberg SP, McCann D, Desai M, Rosenbaum M, Leibel RL, Ferrante AW Jr: Obesity is associated with macrophage accumulation in adipose tissue. J Clin Invest 2003;112: 1796-1808. 
-15 Xu H, Barnes GT, Yang Q, Tan G, Yang D, Chou CJ, Sole J, Nichols A, Ross JS, Tartaglia LA, Chen $\mathrm{H}$ : Chronic inflammation in fat plays a crucial role in the development of obesity-related insulin resistance. J Clin Invest 2003;112:1821-1830.

-16 Cinti S, Mitchell G, Barbatelli G, Murano I, Ceresi E, Faloia E, Wang S, Fortier M, Greenberg AS, Obin MS: Adipocyte death defines macrophage localization and function in adipose tissue of obese mice and humans. J Lipid Res 2005;46:2347-2355.

17 Harman-Boehm I, Bluher M, Redel H, SionVardy N, Ovadia S, Avinoach E, Shai I, Kloting N, Stumvoll M, Bashan N, Rudich A: Macrophage infiltration into omental versus subcutaneous fat across different populations: Effect of regional adiposity and the comorbidities of obesity. J Clin Endocrinol Metab 2007;92:2240-2247.

- 18 Jerschow E, Anwar S, Barzilai N, Rosenstreich D: Macrophages accumulation in visceral and subcutaneous adipose tissue correlates with age. J Allergy Clin Immunol 2007;119(Suppl 1):S179.

19 Kim MJ, Leclercq P, Lanoy E, Cervera P, Antuna-Puente B, Maachi M, Dorofeev E, Slama L, Valantin MA, Costagliola D, Lombes A, Bastard JP, Capeau J: A 6-month interruption of antiretroviral therapy improves adipose tissue function in HIV-infected patients: the ANRS EP29 Lipostop Study. Antivir Ther 2007;12:1273-1283.

20 Sevastianova K, Sutinen J, Kannisto K, Hamsten A, Ristola M, Yki-Jarvinen H: Adipose tissue inflammation and liver fat in patients with highly active antiretroviral therapy-associated lipodystrophy. Am J Physiol Endocrinol Metab 2008;295:E85-E91.

-21 Liu J, Divoux A, Sun J, Zhang J, Clement K, Glickman JN, Sukhova GK, Wolters PJ, Du J, Gorgun CZ, Doria A, Libby P, Blumberg RS, Kahn BB, Hotamisligil GS, Shi GP: Genetic deficiency and pharmacological stabilization of mast cells reduce diet-induced obesity and diabetes in mice. Nat Med 2009;15: 940-945.

-22 Nishimura S, Manabe I, Nagasaki M, Eto K, Yamashita H, Ohsugi M, Otsu M, Hara K, Ueki K, Sugiura S, Yoshimura K, Kadowaki T, Nagai R: Cd8+ effector T cells contribute to macrophage recruitment and adipose tissue inflammation in obesity. Nat Med 2009; 15:914-920.

-23 Winer S, Chan Y, Paltser G, Truong D, Tsui H, Bahrami J, Dorfman R, Wang Y, Zielenski J, Mastronardi F, Maezawa Y, Drucker DJ, Engleman E, Winer D, Dosch HM: Normalization of obesity-associated insulin resistance through immunotherapy. Nat Med 2009;15:921-929.

-24 Feuerer M, Herrero L, Cipolletta D, Naaz A, Wong J, Nayer A, Lee J, Goldfine AB, Benoist C, Shoelson S, Mathis D: Lean, but not obese, fat is enriched for a unique population of regulatory T cells that affect metabolic parameters. Nat Med 2009;15:930-939.
Kintscher U, Hartge M, Hess K, Foryst-Ludwig A, Clemenz M, Wabitsch M, FischerPosovszky P, Barth TF, Dragun D, Skurk T, Hauner $\mathrm{H}$, Bluher $\mathrm{M}$, Unger T, Wolf AM, Knippschild U, Hombach V, Marx N: Tlymphocyte infiltration in visceral adipose tissue: a primary event in adipose tissue inflammation and the development of obesity-mediated insulin resistance. Arterioscler Thromb Vasc Biol 2008;28:1304-1310.

26 Villarroya F, Domingo P, Giralt M: Lipodystrophy in HIV 1-infected patients: lessons for obesity research. Int J Obes (Lond) 2007;31: 1763-1776.

27 Araki S, Okazaki M, Goto S: Impaired lipid metabolism in aged mice as revealed by fasting-induced expression of apolipoprotein mRNAs in the liver and changes in serum lipids. Gerontology 2004;50:206-215.

28 Bays HE, Gonzalez-Campoy JM, Bray GA, Kitabchi AE, Bergman DA, Schorr AB, Rodbard HW, Henry RR: Pathogenic potential of adipose tissue and metabolic consequences of adipocyte hypertrophy and increased visceral adiposity. Expert Rev Cardiovasc Ther 2008;6:343-368.

29 Wu D, Ren Z, Pae M, Guo W, Cui X, Merrill $\mathrm{AH}$, Meydani SN: Aging up-regulates expression of inflammatory mediators in mouse adipose tissue. J Immunol 2007;179: 4829-4839.

30 Guo W, Pirtskhalava T, Tchkonia T, Xie W, Thomou T, Han J, Wang T, Wong S, Cartwright A, Hegardt FG, Corkey BE, Kirkland JL: Aging results in paradoxical susceptibility of fat cell progenitors to lipotoxicity. Am J Physiol Endocrinol Metab 2007;292:E1041E1051.

31 Guo W, Wong S, Xie W, Lei T, Luo Z: Palmitate modulates intracellular signaling, induces endoplasmic reticulum stress, and causes apoptosis in mouse 3T3-L1 and rat primary preadipocytes. Am J Physiol Endocrinol Metab 2007;293:E576-E586.

32 Permana PA, Menge C, Reaven PD: Macrophage-secreted factors induce adipocyte inflammation and insulin resistance. Biochem Biophys Res Commun 2006;341:507-514.

33 Takahashi K, Yamaguchi S, Shimoyama T, Seki H, Miyokawa K, Katsuta H, Tanaka T, Yoshimoto K, Ohno H, Nagamatsu S, Ishida $\mathrm{H}$ : JNK- and IkappaB-dependent pathways regulate MCP-1 but not adiponectin release from artificially hypertrophied 3T3-L1 adipocytes preloaded with palmitate in vitro. Am J Physiol Endocrinol Metab 2008;294: E898-E909.

34 Suganami T, Nishida J, Ogawa Y: A paracrine loop between adipocytes and macrophages aggravates inflammatory changes: role of free fatty acids and tumor necrosis factor alpha. Arterioscler Thromb Vasc Biol 2005;25: 2062-2068.
35 Suganami T, Tanimoto-Koyama K, Nishida J, Itoh M, Yuan X, Mizuarai S, Kotani H, Yamaoka S, Miyake K, Aoe S, Kamei Y, Ogawa Y: Role of the Toll-like receptor 4/NFkappaB pathway in saturated fatty acid-induced inflammatory changes in the interaction between adipocytes and macrophages. Arterioscler Thromb Vasc Biol 2007; 27:84-91.

36 Cartwright MJ, Tchkonia T, Kirkland JL: Aging in adipocytes: potential impact of inherent, depot-specific mechanisms. Exp Gerontol 2007;42:463-471.

37 Kirkland JL, Tchkonia T, Pirtskhalava T, Han J, Karagiannides I: Adipogenesis and aging: does aging make fat go MAD? Exp Gerontol 2002;37:757-767.

- 38 Kirkland JL, Hollenberg CH, Gillon WS: Age, anatomic site, and the replication and differentiation of adipocyte precursors. Am J Physiol 1990;258:C206-C210.

39 Karagiannides I, Tchkonia T, Dobson DE, Steppan CM, Cummins P, Chan G, Salvatori K, Hadzopoulou-Cladaras M, Kirkland JL: Altered expression of C/EBP family members results in decreased adipogenesis with aging. Am J Physiol Regul Integr Comp Physiol 2001;280:R1772-R1780.

40 Bertrand HA, Lynd FT, Masoro EJ, Yu BP: Changes in adipose mass and cellularity through the adult life of rats fed ad libitum or a life-prolonging restricted diet. J Gerontol 1980;35:827-835.

-41 Spalding KL, Arner E, Westermark PO, Bernard S, BuchholzBA, Bergmann O, Blomqvist L, Hoffstedt J, Naslund E, Britton T, Concha H, Hassan M, Ryden M, Frisen J, Arner P: Dynamics of fat cell turnover in humans. Nature 2008;453:783-787.

- 42 Hauner H, Entenmann G, Wabitsch M, Gaillard D, Ailhaud G, Negrel R, Pfeiffer EF: Promoting effect of glucocorticoids on the differentiation of human adipocyte precursor cells cultured in a chemically defined medium. J Clin Invest 1989;84:1663-1670.

43 Tang QQ, Zhang JW, Daniel Lane M: Sequential gene promoter interactions of $\mathrm{c} /$ ebpbeta, c/ebpalpha, and ppargamma during adipogenesis. Biochem Biophys Res Commun 2004;319:235-239.

44 Karagiannides I, Thomou T, Tchkonia T, Pirtskhalava T, Kypreos KE, Cartwright A, Dalagiorgou G, Lash TL, Farmer SR, Timchenko NA, Kirkland JL: Increased CUG triplet repeat-binding protein-1 predisposes to impaired adipogenesis with aging. J Biol Chem 2006;281:23025-23033.

45 Tchkonia T, Pirtskhalava T, Thomou T, Cartwright MJ, Wise B, Karagiannides I, Shpilman A, Lash TL, Becherer JD, Kirkland JL: Increased TNFalpha and CCAAT/enhancer-binding protein homologous protein with aging predispose preadipocytes to resist adipogenesis. Am J Physiol Endocrinol Metab 2007;293:E1810-E1819. 
-46 Hotamisligil GS: Molecular mechanisms of insulin resistance and the role of the adipocyte. Int J Obes Relat Metab Disord 2000; 24(Suppl 4):S23-S27.

-47 Tchkonia T, Lenburg M, Thomou T, Giorgadze N, Frampton G, Pirtskhalava T, Cartwright A, Cartwright M, Flanagan J, Karagiannides I, Gerry N, Forse RA, Tchoukalova Y, Jensen MD, Pothoulakis C, Kirkland JL: Identification of depot-specific human fat cell progenitors through distinct expression profiles and developmental gene patterns. Am J Physiol Endocrinol Metab 2007;292: E298-E307.

-48 Gesta S, Bluher M, Yamamoto Y, Norris AW, Berndt J, Kralisch S, Boucher J, Lewis C, Kahn CR: Evidence for a role of developmental genes in the origin of obesity and body fat distribution. Proc Natl Acad Sci USA 2006; 103:6676-6681.

49 Tchkonia T, Giorgadze N, Pirtskhalava T, Thomou T, DePonte M, Koo A, Forse RA, Chinnappan D, Martin-Ruiz C, von Zglinicki T, Kirkland JL: Fat depot-specific characteristics are retained in strains derived from single human preadipocytes. Diabetes 2006;55:2571-2578.
50 Van Harmelen V, Rohrig K, Hauner H: Comparison of proliferation and differentiation capacity of human adipocyte precursor cells from the omental and subcutaneous adipose tissue depot of obese subjects. Metabolism 2004;53:632-637.

51 Chung S, Lapoint K, Martinez K, Kennedy A, Boysen Sandberg M, McIntosh MK: Preadipocytes mediate lipopolysaccharide-induced inflammation and insulin resistance in primary cultures of newly differentiated human adipocytes. Endocrinology 2006; 147:5340-5351.

52 Harkins JM, Moustaid-Moussa N, Chung YJ, Penner KM, Pestka JJ, North CM, Claycombe KJ: Expression of interleukin-6 is greater in preadipocytes than in adipocytes of 3T3-L1 cells and C57BL/6J and ob/ob mice. J Nutr 2004;134:2673-2677.

53 Vitseva OI, Tanriverdi K, Tchkonia TT, Kirkland JL, McDonnell ME, Apovian CM, Freedman J, Gokce N: Inducible toll-like receptor and NF-kappaB regulatory pathway expression in human adipose tissue. Obesity (Silver Spring) 2008;16:932-937.

54 Murano I, Barbatelli G, Parisani V, Latini C, Muzzonigro G, Castellucci M, Cinti S: Dead adipocytes, detected as crown-like structures, are prevalent in visceral fat depots of genetically obese mice. J Lipid Res 2008;49: 1562-1568.
55 Sebastian CL, Celada JA: Molecular and cellular aspects of macrophage aging; in Fulop T, Franceschi C, Hirokawa K, Pawelec G (eds): Handbook on Immunosenescence, Dordrecht, Springer Science \& Business Media B.V., 2009, pp 919-945.

56 Tchkonia T, Giorgadze N, Pirtskhalava T, Thomou T, Villaret A, Bouloumie A, von Zglinicki T, Kirkland JL: Cellular senescence and inflammation in obesity. Obesity (Supplement) 2009;17:S57.

57 Minamino T, Orimo M, Shimizu I, Kunieda T, Yokoyama M, Ito T, Nojima A, Nabetani A, Oike Y, Matsubara H, Ishikawa F, Komuro I: A crucial role for adipose tissue p53 in the regulation of insulin resistance. Nat Med 2009; 15:1082-1087.

58 Baker DJ, Perez-Terzic C, Jin F, Pitel K, Niederlander NJ, Jeganathan K, Yamada S, Reyes S, Rowe L, Hiddinga HJ, Eberhardt NL, Terzic A, van Deursen JM: Opposing roles for p16Ink4a and p19Arf in senescence and ageing caused by BubR1 insufficiency. Nat Cell Biol 2008;10:825-836. 